Bull. Austral. Math. Soc.

$47 \mathrm{~B} 47,47 \mathrm{~L} 10,47 \mathrm{~B} 49$

VOL. $66(2002) \quad[477-486]$

\title{
DERIVATIONS, LOCAL DERIVATIONS AND ATOMIC BOOLEAN SUBSPACE LATTICES
}

\author{
Pengtong Li and Jipu Ma
}

\begin{abstract}
Let $\mathcal{L}$ be an atomic Boolean subspace lattice on a Banach space $X$. In this paper, we prove that if $\mathcal{M}$ is an ideal of $\operatorname{Alg} \mathcal{L}$ then every derivation $\delta$ from $\operatorname{Alg} \mathcal{L}$ into $\mathcal{M}$ is necessarily quasi-spatial, that is, there exists a densely defined closed linear operator $T: \mathcal{D}(T) \subseteq X \rightarrow X$ with its domain $\mathcal{D}(T)$ invariant under every element of $\operatorname{Alg} \mathcal{L}$, such that $\delta(A) x=(T A-A T) x$ for every $A \in \operatorname{Alg} \mathcal{L}$ and every $x \in \mathcal{D}(T)$. Also, if $\mathcal{M} \subseteq \mathcal{B}(X)$ is an $\operatorname{Alg} \mathcal{L}$-module then it is shown that every local derivation from $\operatorname{Alg} \mathcal{L}$ into $\mathcal{M}$ is necessary a derivation. In particular, every local derivation from $\operatorname{Alg} \mathcal{L}$ into $\mathcal{B}(X)$ is a derivation and every local derivation from $\operatorname{Alg} \mathcal{L}$ into itself is a quasi-spatial derivation.
\end{abstract}

\section{INTRODUCTION}

Let $\mathcal{A}$ be an (associative) algebra, $\mathcal{M}$ be an $\mathcal{A}$-module, and $\delta: \mathcal{A} \rightarrow \mathcal{M}$ be a linear map. Recall that $\delta$ is a derivation if $\delta(a b)=\delta(a) b+a \delta(b)$ holds for all $a$ and $b$ in $\mathcal{A}$, and that $\delta$ is an inner derivation if there exists an element $m$ in $\mathcal{M}$ such that $\delta(a)=m a-a m$ holds for all $a$ in $\mathcal{A}$. Also, $\delta$ is a local derivation (respectively, local inner derivation) if for each $a$ in $\mathcal{A}$ there is a derivation (respectively, inner derivation) $\delta_{a}$ from $\mathcal{A}$ into $\mathcal{M}$ depending on $a$, such that $\delta(a)=\delta_{a}(a)$.

The concept of local derivations was first introduced by Kadison (see [6]) who proved that if $\mathcal{A}$ is a von Neumann algebra and $\mathcal{M}$ is a dual $\mathcal{A}$-module, then all norm-continuous local derivations from $\mathcal{A}$ into $\mathcal{M}$ are in fact derivations. In recent years, there has been a growing interest in the study of local derivations of operator algebras (see $[2,3,4,5,11])$. If $X$ is a Banach space, as usual, we use $\mathcal{B}(X)$ to denote the algebra of all bounded linear operators on $X$. In [11], Larson and Sourour proved that all derivations and all local inner derivations from $\mathcal{B}(X)$ into itself are inner derivations (hence, every local derivation from $\mathcal{B}(X)$ into itself is a derivation). Jing in [3] generalised Larson-Sourour's results. He proved that if $\mathcal{A}$ is a reflexive operator algebra on a Banach space $X$ such that both $0_{+} \neq 0$ and $X_{-} \neq X$ in Lat $\mathcal{A}$, then every derivation $\delta: \mathcal{A} \rightarrow \mathcal{A}$ is spatial, that is, there

Received 8th May, 2002

Supported by the National Natural Science Foundation of People's Republic China (Grant No. 19971039). The authors would like to thank the referee for many helpful comments.

Copyright Clearance Centre, Inc. Serial-fee code: 0004-9727/02 \$A2.00+0.00. 
exists a $T \in \mathcal{B}(X)$ such that $\delta(A)=T A-A T$ for all $A \in \mathcal{A}$, and all norm-continuous local inner derivations from $\mathcal{A}$ into $\mathcal{A}$ are inner derivations when $X$ is additionally reflexive. Moreover, in a recent paper (see [4]), he has shown that every local derivation from $\mathcal{A}$ into itself is a derivation.

Let $\mathcal{L}_{1}$ and $\mathcal{L}_{2}$ be finite distributive subspace lattices on the Banach spaces $X_{1}$ and $X_{2}$, respectively. It was shown by Oreste Panaia in [13] that every rank-preserving algebraic isomorphism $\phi$ of $\operatorname{Alg} \mathcal{L}_{1}$ onto $\operatorname{Alg} \mathcal{L}_{2}$ is quasi-spatial, which means that there exists a closed, densely defined, injective linear transformation $T: \mathcal{D}(T) \subset X_{1} \rightarrow X_{2}$ with dense range, and with its domain $\mathcal{D}(T)$ invariant under every element of $\operatorname{Alg} \mathcal{L}_{1}$, such that $\phi(A) T x=T A x$, for every $x \in \mathcal{D}(T)$ and every $A \in A \lg \mathcal{L}_{1}$. Recently, Katavolos, Lambrou and Longstaff proved that every algebraic isomorphism between $\operatorname{Alg} \mathcal{P}_{1}$ and $\operatorname{Alg} \mathcal{P}_{2}$ is also quasi-spatial, where $\mathcal{P}_{1}$ and $\mathcal{P}_{2}$ are two pentagon subspace lattices on Banach spaces (see [7]). In addition, we know from $[7,13]$ that the notion of quasi-spatiality of algebraic isomorphisms of operator algebras was introduced by Lambrou in [9], where it is proved that the algebraic isomorphisms from $\operatorname{Alg} \mathcal{L}_{1}$ onto $\operatorname{Alg} \mathcal{L}_{2}$ are quasi-spatial for any pair $\mathcal{L}_{1}, \mathcal{L}_{2}$ of atomic Boolean subspace lattices on Banach spaces, but we can not find this reference

Here, motivated by the results mentioned above, we consider the derivations and the local derivations of the reflexive operator algebras on Banach spaces with atomic Boolean invariant subspace lattices. For the statements of our results we give the following definition concerning quasi-spatiality of derivations of operator algebras, which seems to be known.

DEFinition 1.1: Let $X$ be a Banach space, $\mathcal{A} \subseteq \mathcal{B}(X)$ be a subalgebra, and $\mathcal{M}$ $\subseteq \mathcal{B}(X)$ be an $\mathcal{A}$-module. A derivation $\delta: \mathcal{A} \rightarrow \mathcal{M}$ is called quasi-spatial if there exists a densely defined closed linear operator $T: \mathcal{D}(T) \subseteq X \rightarrow X$ with its domain $\mathcal{D}(T)$ invariant under every element of $\mathcal{A}$, such that $\delta(A) x=(T A-A T) x$ holds for every $A \in \mathcal{A}$ and every $x \in \mathcal{D}(T)$.

Let $\mathcal{L}$ be an atomic Boolean subspace lattices on a Banach space $X$. We shall show that if $\mathcal{M}$ is an ideal of $\operatorname{Alg} \mathcal{L}$ then every derivation $\delta$ from $\operatorname{Alg} \mathcal{L}$ into $\mathcal{M}$ is necessarily quasi-spatial. Also, if $\mathcal{M} \subseteq \mathcal{B}(X)$ is an $\operatorname{Alg} \mathcal{L}$-module then it is shown that every local derivation from $\operatorname{Alg} \mathcal{L}$ into $\mathcal{M}$ is necessary a derivation. In particular, every local derivation from $\operatorname{Alg} \mathcal{L}$ into $\mathcal{B}(X)$ is a derivation and every local derivation from $\operatorname{Alg} \mathcal{L}$ into itself is a quasi-spatial derivation.

\section{Notation AND PRELIMINARIES}

Throughout $X$ will denote a fixed real or complex Banach space, with topological dual $X^{*}$. The terms operator acting on $X$ and subspace of $X$ will mean bounded linear map of $X$ into itself and norm-closed linear manifold of $X$, respectively. For $T \in \mathcal{B}(X)$, denote by $T^{*}$ the adjoint of $T$, by $G(T)$ the graph of $T$, that is $G(T)=\{(x, T x): x \in X\}$, 
and by $I$ the identity operator on $X$. For $x \in X$ and $f^{*} \in X^{*}$, the operator $x \otimes f^{*}$ is defined by $y \mapsto f^{*}(y) x$ for $y \in X$. This operator has rank one if and only if both $x$ and $f^{*}$ are nonzero. Note that the adjoint of $x \otimes f^{*}$ is the operator $f^{*} \otimes \widehat{x}$ which is given by $g^{*} \mapsto g^{*}(x) f^{*}$ for $g^{*} \in X^{*}$, where $\widehat{x}$ is the image of $x$ under the canonical map of $X$ into $X^{* *}$ (the second dual of $X$ ). For any non-empty subset $L \subseteq X, L^{\perp}$ denotes its annihilator, that is, $L^{\perp}=\left\{f^{*} \in X^{*}: f^{*}(x)=0\right.$ for all $\left.x \in L\right\}$; and dually, for any non-empty subset $F \subseteq X^{*}, F_{\perp}$ denotes its pre-annihilator, that is, $F_{\perp}=\{x$ $\in X: f^{*}(x)=0$ for all $\left.f^{*} \in F\right\}$. For every family $\left\{L_{\gamma}\right\}_{\gamma \in \Gamma}$ of subspaces of $X$, we have $\left(\bigvee_{\gamma \in \Gamma} L_{\gamma}\right)^{\perp}=\bigcap_{\gamma \in \Gamma} L_{\gamma}^{\perp}$ and $\bigvee_{\gamma \in \Gamma} L_{\gamma}^{\perp} \subseteq\left(\bigcap_{\gamma \in \Gamma} L_{\gamma}\right)^{\perp}$; indeed, it is easy to verify that $\left(\bigcap_{\gamma \in \Gamma} L_{\gamma}\right)^{\perp}$ is the weak star closure of $\bigvee_{\gamma \in \Gamma} L_{\gamma}^{\perp}$. Here ' $V$ ' and ' $N$ ' denote 'norm-closed linear span' and 'set theoretic intersection'.

If $\mathcal{L}$ is a family of subspaces of $X$, we say that $\mathcal{L}$ is a subspace lattice on $X$ if it contains ( 0 ) and $X$, and is closed under the operations $\vee$ and $\cap$, that is, for any family $\left\{L_{\gamma}\right\}_{\gamma \in \Gamma}$ of elements of $\mathcal{L}, \bigvee_{\gamma \in \Gamma} L_{\gamma} \in \mathcal{L}$ and $\bigcap_{\gamma \in \Gamma} L_{\gamma} \in \mathcal{L}$. For any family $\mathcal{F}$ of subspaces of $X$, let Alg $\mathcal{F}$ denote the algebra of all operators on $X$ which leave every subspace in $\mathcal{F}$ invariant. Dually, for any family $\mathcal{A}$ of operators on $X$, let Lat $\mathcal{A}$ denote the set of all subspaces of $X$ which are invariant under every operator in $\mathcal{A}$. It is clear that $\operatorname{Alg} \mathcal{F}$ is a unital weakly closed operator algebra and Lat $\mathcal{A}$ is a subspace lattice. We say that a subspace lattice $\mathcal{L}$ is reflexive if $\mathcal{L}=\operatorname{Lat} A \lg \mathcal{L}$, and an operator algebra $\mathcal{A}$ is reflexive if $\mathcal{A}=\operatorname{AlgLat} \mathcal{A}$.

A subspace lattice $\mathcal{L}$ is called complemented if for every $L \in \mathcal{L}$ there is an element $L^{\prime} \in \mathcal{L}$, a lattice complement of $L$, such that $L \vee L^{\prime}=X$ and $L \cap L^{\prime}=(0)$, and distributive if the identity $L \cap(M \vee N)=(L \cap M) \vee(L \cap N)$ and its dual hold for all $L, M, N \in \mathcal{L}$. A nonzero complemented and distributive subspace lattice is called a Boolean subspace lattice. An nonzero element $K$ in a subspace lattice $\mathcal{L}$ is called an atom if, whenever $L \in \mathcal{L}$ such that $(0) \subseteq L \subseteq K$, then either $L=(0)$ or $L=K$. A subspace lattice $\mathcal{L}$ is called atomic if each element of $\mathcal{L}$ is the closed linear span of the atoms it contains. It is well known that an atomic Boolean subspace lattice is completely distributive (see $[\mathbf{8}, \mathbf{1 2}]$ ). From [12] we know that a subspace lattice is completely distributive if and only if it is strongly reflexive. For the standard definition concerning completely distributive subspace lattices and the alternative characterisations see $[8,12]$. From these two references we can find the following lemma which is crucial to this paper.

Lemma 2.1. If $\mathcal{L}$ is a subspace lattice on $X$, then the rank one operator $x \otimes$ $f^{*} \in \operatorname{Alg} \mathcal{L}$ if and only if there exists a $L \in \mathcal{L}$ such that $x \in L$, and $f^{*} \in L_{-}^{\perp}$, where $L_{-}=\bigvee\{M \in \mathcal{L}: M \nsupseteq L\}$ and $L_{-}^{\perp}$ means $\left(L_{-}\right)^{\perp}$.

In the above lemma, if $\mathcal{L}$ is an atomic Boolean subspace lattice, then the subspace $L$ can be taken to be an atom of $\mathcal{L}$ since a nonzero $K \in \mathcal{L}$ is an atom if and only if $K_{-} \neq \neq X$ (see [12]), in which case $L_{-}$is the same as $L^{\prime}$ (see [12]), the (unique) lattice complement 
of $L$. It is worth noting that the equations $(0)_{+}=(0)$ and $X_{-}=X$ are always true in an atomic Boolean subspace lattice $\mathcal{L}$, so the associated reflexive algebra $\operatorname{Alg} \mathcal{L}$ is not in the class of reflexive algebras introduced in $[3,4]$. Here $(0)_{+}=\bigcap\{L \in \mathcal{L}: L \neq(0)\}$.

Finally, for an atomic Boolean subspace lattice $\mathcal{L}$ on $X, \mathcal{L}_{A}$ will denote the set of atoms of $\mathcal{L}, \mathcal{L}_{A}^{\perp}$ the set $\left\{K_{-}^{\perp}: K \in \mathcal{L}_{A}\right\}$ and $\mathcal{R}_{\mathcal{L}}$ the set of rank one operators in $\operatorname{Alg} \mathcal{L}$. Also, let $\left\langle\mathcal{L}_{A}\right\rangle$ and $\left\langle\mathcal{L}_{A}^{\perp}\right\rangle$ be the (not necessarily closed) linear span of $\mathcal{L}_{A}$ and $\mathcal{L}_{A}^{\perp}$, respectively.

\section{QUASI-SPATIALITY OF DERIVATIONS}

Throughout this section, $\mathcal{L}$ will be an atomic Boolean subspace lattice on the Banach space $X, \mathcal{M}$ be an ideal of $\operatorname{Alg} \mathcal{L}$, and $\delta: \operatorname{Alg} \mathcal{L} \rightarrow \mathcal{M}$ be a derivation. The main result of this section shows that $\delta$ is quasi-spatial.

Lemma 3.1. For every $K \in \mathcal{L}_{A}$, there exist two linear maps $T_{K}: K \rightarrow K$ and $S_{K}: K_{-}^{\perp} \rightarrow K_{-}^{\perp}$, such that

(i) $\delta(A) x=\left(T_{K} A-A T_{K}\right) x$, for $A \in \operatorname{Alg} \mathcal{L}$ and $x \in K$;

(ii) $\delta(A)^{*} f^{*}=\left(S_{K} A^{*}-A^{*} S_{K}\right) f^{*}$, for $A \in \operatorname{Alg} \mathcal{L}$ and $f^{*} \in K_{-}^{\perp}$;

(iii) $\delta\left(x \otimes f^{*}\right)=T_{K} x \otimes f^{*}+x \otimes S_{K} f^{*}$, for $x \in K$ and $f^{*} \in K_{-}^{\perp}$.

Proof: Since $K \cap K_{-}=(0)$, we can choose fixed nonzero elements $x_{K} \in K$, $f_{K}^{*} \in K_{\perp}^{\perp}$ with $f_{K}^{*}\left(x_{K}\right)=1$.

(i) By Lemma 2.1, $x \otimes f_{K}^{*} \in \operatorname{Alg} \mathcal{L}$ for any $x \in K$. Then define a map $T_{K}$ by

$$
T_{K} x=\delta\left(x \otimes f_{K}^{*}\right) x_{K}, \quad x \in K .
$$

Clearly, $T_{K}$ is linear. Since $\mathcal{M}$ is an ideal of $\operatorname{Alg} \mathcal{L}$ and $x_{K} \in K$, we have $T_{K} K \subseteq K$. For $A \in \operatorname{Alg} \mathcal{L}$ and $x \in K$, then $A x \in K$ and

$$
\delta\left(A x \otimes f_{K}^{*}\right)=\delta\left(A \cdot x \otimes f_{K}^{*}\right)=\delta(A) \cdot x \otimes f_{K}^{*}+A \cdot \delta\left(x \otimes f_{K}^{*}\right) .
$$

By letting the two sides of the above equation act on $x_{K}$, we obtain $\delta(A) x=\left(T_{K} A\right.$ $\left.-A T_{K}\right) x$.

(ii) The proof is a dual version of (i). For all $f^{*} \in K_{-}^{\perp}$ we have $x_{K} \otimes f^{*} \in \operatorname{Alg} \mathcal{L}$. Define a linear map $S_{K}$ by

$$
S_{K} f^{*}=\delta\left(x_{K} \otimes f^{*}\right)^{*} f_{K}^{*}, \quad f^{*} \in K_{-}^{\perp} .
$$

That $S_{K} K_{-}^{\perp} \subseteq K_{-}^{\perp}$ follows from the fact that $A^{*} K_{-}^{\perp} \subseteq K_{-}^{\perp}$ holds for all $A \in \operatorname{Alg} \mathcal{L}$. For $A \in \operatorname{Alg} \mathcal{L}$ and $f^{*} \in K_{-}^{\perp}$, we have $\delta\left(x_{K} \otimes A^{*} f^{*}\right)=\delta\left(x_{K} \otimes f^{*} \cdot A\right)=\delta\left(x_{K} \otimes f^{*}\right) A+x_{K} \otimes$ $f^{*} \cdot \delta(A)$. Taking the adjoint for each operator in this equation, then

$$
\delta\left(x_{K} \otimes A^{*} f^{*}\right)^{*}=A^{*} \delta\left(x_{K} \otimes f^{*}\right)^{*}+\delta(A)^{*}\left(f^{*} \otimes \widehat{x_{K}}\right) .
$$


By applying the above equation to $f_{K}^{*}$, we obtain $\delta(A)^{*} f^{*}=\left(S_{K} A^{*}-A^{*} S_{K}\right) f^{*}$.

(iii) For $x \in K$ and $f^{*} \in K_{-}^{\perp}$, we have by the definitions of $T_{K}$ and $S_{K}$

$$
\begin{aligned}
\delta\left(x \otimes f^{*}\right) & =\delta\left(x \otimes f_{K}^{*} \cdot x_{K} \otimes f^{*}\right) \\
& =\delta\left(x \otimes f_{K}^{*}\right) \cdot x_{K} \otimes f^{*}+x \otimes f_{K}^{*} \cdot \delta\left(x_{K} \otimes f^{*}\right) \\
& =\delta\left(x \otimes f_{K}^{*}\right) x_{K} \otimes f^{*}+x \otimes \delta\left(x_{K} \otimes f^{*}\right)^{*} f_{K}^{*} \\
& =T_{K} x \otimes f^{*}+x \otimes S_{K} f^{*}
\end{aligned}
$$

This completes the proof.

For the remainder of this section, for any given $K \in \mathcal{L}_{A}, T_{K}$ and $S_{K}$ will denote the linear maps as constructed in Lemma 3.1. Obviously, they depend on the choices made for $x_{K}$ and $f_{K}^{*}$, so it will be assumed that those choices have been made for each $K \in \mathcal{L}_{A}$.

Suppose that $K_{1}, \cdots, K_{n}$ are distinct atoms of $\mathcal{L}$, and $x_{i} \in K_{i}, f_{i}^{*} \in K_{i-}^{1}$ for $i=1, \cdots, n$. Then, from $\sum_{i=1}^{n} x_{i}=0$ and $\sum_{i=1}^{n} f_{i}^{*}=0$ we can obtain every $x_{i}$ and every $f_{i}^{*}$ is zero, respectively. For example, let $\sum_{i=1}^{n} f_{i}^{*}=0$. For any $f_{i}^{*}$, we have by complete distributivity and De Morgans' laws (see $[\mathbf{1}$, p. 5])

$$
\begin{aligned}
f_{i}^{*} & \in K_{i-}^{\perp} \cap\left(\bigvee_{j \neq i} K_{j-}^{\perp}\right) \subseteq K_{i-}^{\perp} \cap\left(\bigcap_{j \neq i} K_{j-}\right)^{\perp}=\left(K_{i-} \vee\left(\bigcap_{j \neq i} K_{j-}\right)\right)^{\perp} \\
& =\left(K_{i}^{\prime} \vee\left(\bigcap_{j \neq i} K_{j}^{\prime}\right)\right)^{\perp}=\left(K_{i}^{\prime} \vee\left(\bigvee_{j \neq i} K_{j}\right)^{\prime}\right)^{\perp}=\left(\left(K_{i} \cap\left(\bigvee_{j \neq i} K_{j}\right)\right)^{\prime}\right)^{\perp} \\
& =\left(\left(\bigvee_{j \neq i}\left(K_{i} \cap K_{j}\right)\right)^{\prime}\right)^{\perp}=\left((0)^{\prime}\right)^{\perp}=X^{\perp}=(0),
\end{aligned}
$$

and so $f_{i}^{*}=0$, as desired. Thus every $x \in\left\langle\mathcal{L}_{A}\right\rangle$ has a representation as follows: $x=\sum_{i=1}^{n} x_{i}$ with $x_{i} \in K_{i}, 1 \leqslant i \leqslant n$, where $K_{1}, \cdots, K_{n}$ are distinct atoms of $\mathcal{L}$. If $x$ is nonzero and each $x_{i}$ is required to be nonzero, this representation is unique up to permutations of the atoms. Similar statements can apply to the elements of $\left\langle\mathcal{L}_{A}^{\perp}\right\rangle$. Therefore, the linear maps $T_{0}$ and $S_{0}$ in the following definition are well-defined.

Definition 3.1: Define $T_{0}:\left\langle\mathcal{L}_{A}\right\rangle \rightarrow\left\langle\mathcal{L}_{A}\right\rangle$ by $T_{0} x=\sum_{i=1}^{m} T_{K_{i}} x_{i}$, where $x=\sum_{i=1}^{m} x_{i}$ with $x_{i} \in K_{i}, 1 \leqslant i \leqslant m$, and $K_{1}, \cdots, K_{m}$ being distinct atoms of $\mathcal{L}$; and define $S_{0}:\left\langle\mathcal{L}_{A}^{\perp}\right\rangle \rightarrow\left\langle\mathcal{L}_{A}^{\perp}\right\rangle$ by $S_{0} f^{*}=\sum_{j=1}^{n} S_{L_{j}} f_{j}^{*}$, where $f^{*}=\sum_{j=1}^{n} f_{j}^{*}$ with $f_{j}^{*} \in L_{j-}^{\perp}, 1 \leqslant j \leqslant n$, and $L_{1}, \cdots, L_{n}$ being distinct atoms of $\mathcal{L}$.

Lemma 3.2. For $A \in \operatorname{Alg} \mathcal{L}$ and $x \in\left\langle\mathcal{L}_{A}\right\rangle$, we have $\delta(A) x=\left(T_{0} A-A T_{0}\right) x$.

PROOF: It is a routine computation by the definition of $T_{0}$ and Lemma 3.1 (i).

LEMmA 3.3. For $x \in\left\langle\mathcal{L}_{A}\right\rangle$ and $f^{*} \in\left\langle\mathcal{L}_{A}^{\perp}\right\rangle$, we have $f^{*}\left(T_{0} x\right)+\left(S_{0} f^{*}\right)(x)=0$. 
Proof: We first prove that $f^{*}\left(T_{0} x\right)+\left(S_{0} f^{*}\right)(x)=0$ holds for $x \in K$ and $f^{*} \in K_{-}^{\perp}$, where $K \in \mathcal{L}_{A}$ is arbitrary. For, assume that $x$ and $f^{*}$ are both nonzero. By Lemma 3.1 (iii) we have

$$
\delta\left(\left(x \otimes f^{*}\right)^{2}\right)=f^{*}(x) \delta\left(x \otimes f^{*}\right)=f^{*}(x) T_{0} x \otimes f^{*}+f^{*}(x) x \otimes S_{0} f^{*}
$$

on the other hand,

$$
\begin{aligned}
\delta\left(\left(x \otimes f^{*}\right)^{2}\right) & =\delta\left(x \otimes f^{*}\right) \cdot x \otimes f^{*}+x \otimes f^{*} \cdot \delta\left(x \otimes f^{*}\right) \\
& =f^{*}(x) T_{0} x \otimes f^{*}+\left(S_{0} f^{*}\right)(x) x \otimes f^{*}+f^{*}\left(T_{0} x\right) x \otimes f^{*}+f^{*}(x) x \otimes S_{0} f^{*}
\end{aligned}
$$

Hence $f^{*}\left(T_{0} x\right)+\left(S_{0} f^{*}\right)(x)=0$.

For the general case, combine what has just been shown with the fact that if $x \in K$ and $f^{*} \in L_{-}^{\perp}$, where $K, L$ are distinct atoms of $\mathcal{L}$, then $f^{*}\left(T_{K} x\right)=0$ and $\left(S_{L} f^{*}\right)(x)=0$ since $K \subseteq L_{-}$. A direct computation gives the required result, and we are done.

LEMmA 3.4. There exists a linear map $T: \mathcal{D}(T) \subseteq X \rightarrow X$ which is the extension of $T_{0}$, such that the norm-closure $\overline{G\left(T_{0}\right)}$ of the graph of $T_{0}$ is the graph $G(T)$ of $T$ and $\overline{\mathcal{D}(T)}=X$.

Proof: To define $T$ we let $\mathcal{D}=\left\{x \in X:(x, y) \in \overline{G\left(T_{0}\right)}\right.$, for some $\left.y \in X\right\}$. Then $\mathcal{D}$ is obviously a linear manifold. Since $\mathcal{L}$ is an atomic Boolean subspace lattice, $\left\langle\mathcal{L}_{A}\right\rangle$ is dense in $X$. Hence, since $\left\langle\mathcal{L}_{A}\right\rangle \subseteq \mathcal{D}, \mathcal{D}$ is dense in $X$.

For any $x \in \mathcal{D}$, we shall show that there exists a unique $y \in X$, such that $(x, y)$ $\in \overline{G\left(T_{0}\right)}$. For, assume that $\left(x, y_{1}\right),\left(x, y_{2}\right) \in \overline{G\left(T_{0}\right)}$ with $y_{1}, y_{2} \in X$. We then have $\left(0, y_{1}\right.$ $\left.-y_{2}\right) \in \overline{G\left(T_{0}\right)}$. Thus, there is a sequence $\left\{x_{n}\right\}_{1}^{\infty}$ of elements of $\left\langle\mathcal{L}_{A}\right\rangle$, such that $x_{n} \rightarrow 0$ and $T_{0} x_{n} \rightarrow y_{1}-y_{2}$. For any $f^{*} \in\left\langle\mathcal{L}_{A}^{\frac{1}{A}}\right\rangle$, it follows that $f^{*}\left(T_{0} x_{n}\right) \rightarrow f^{*}\left(y_{1}-y_{2}\right)$. On the other hand, from Lemma 3.3 we have $f^{*}\left(T_{0} x_{n}\right)+\left(S_{0} f^{*}\right)\left(x_{n}\right)=0$ for all $n$. Thus $f^{*}\left(T_{0} x_{n}\right) \rightarrow 0$ since $\left(S_{0} f^{*}\right)\left(x_{n}\right) \rightarrow 0$, and so $f^{*}\left(y_{1}-y_{2}\right)=0$. Thus $y_{1}=y_{2}$ once the fact that $\left\langle\mathcal{L}_{A}^{\frac{1}{A}}\right\rangle$ is weak star dense in $X^{*}$ is proved to be true. Indeed, from $[8,12]$ we know that

$$
\begin{aligned}
(0) & =\bigcap\left\{K_{-}: K \in \mathcal{L} \text { and } K \neq(0)\right\} \\
& =\bigcap\left\{K_{-}: K \in \mathcal{L}, K \neq(0) \text { and } K_{-} \neq X\right\} \\
& =\bigcap\left\{K_{-}: K \in \mathcal{L} \text { an atom }\right\} .
\end{aligned}
$$

As remarked in the first paragraph of Section 2, the annihilator of the last expression above is the weak star closure of $\left\langle\mathcal{L}_{A}^{\frac{1}{A}}\right\rangle$, as desired.

Up till this point we can define a map $T: \mathcal{D}(T) \subseteq X \rightarrow X$ in an obvious way, such that $G(T)=\overline{G\left(T_{0}\right)}$, where $\mathcal{D}(T)=\mathcal{D}$. Clearly, $T$ is linear and extends $T_{0}$. The proof is complete.

Now we are in a position to prove the main result of this section. 
THEOREM 3.1. Let $\mathcal{L}$ be an atomic Boolean subspace lattice on $X$, and $\mathcal{M}$ be an ideal of $\operatorname{Alg} \mathcal{L}$. Then every derivation $\delta$ from $\operatorname{Alg} \mathcal{L}$ into $\mathcal{M}$ is necessarily quasi-spatial.

Proof: Let $T$ be as in Lemma 3.4. Obviously, $T$ is closed and densely defined. It remains to show that $\mathcal{D}(T)$ is invariant under every element of $\operatorname{Alg} \mathcal{L}$ and $\delta(A) x$ $=(T A-A T) x$, for every $A \in \operatorname{Alg} \mathcal{L}$ and every $x \in \mathcal{D}(T)$.

Let $A \in \operatorname{Alg} \mathcal{L}$ and $x \in \mathcal{D}(T)$. Then $(x, T x) \in \overline{G\left(T_{0}\right)}$ by Lemma 3.4. Thus there exists a sequence $\left\{x_{n}\right\}_{1}^{\infty}$ of elements of $\left\langle\mathcal{L}_{A}\right\rangle$, such that $x_{n} \rightarrow x$ and $T_{0} x_{n} \rightarrow T x$. Moreover, we have that $A x_{n} \rightarrow A x, A T_{0} x_{n} \rightarrow A T x$ and $\delta(A) x_{n} \rightarrow \delta(A) x$. From Lemma 3.2 it follows that $\delta(A) x_{n}=\left(T_{0} A-A T_{0}\right) x_{n}$ for every $n$, and so $T_{0} A x_{n} \rightarrow \delta(A) x+A T x$. We therefore obtain that $\left(A x_{n}, T_{0} A x_{n}\right) \rightarrow(A x, \delta(A) x+A T x)$. Since $\left(A x_{n}, T_{0} A x_{n}\right) \in G\left(T_{0}\right)$, $(A x, \delta(A) x+A T x) \in G(T)$. Hence $A x \in \mathcal{D}(T)$ which means $A \mathcal{D}(T) \subseteq \mathcal{D}(T)$, and $T A x=\delta(A) x+A T x$, that is $\delta(A) x=(T A-A T) x$. This completes the proof.

Remark 3.1. In this section, the condition that $\mathcal{M}$ is an ideal of $\operatorname{Alg} \mathcal{L}$ is mainly used to guarantee the validity of Lemma 3.3. In addition, $\mathcal{M}$ may be equal to $\operatorname{Alg} \mathcal{L}$.

REMARK 3.2. Note that $T$ (as in Lemma 3.4) need not be injective and have dense range. For example, if $\delta: \operatorname{Alg} \mathcal{L} \rightarrow \mathcal{M}$ is the zero map, then $\mathcal{D}(T)=X$ and $T=0$.

\section{LOCAL DERIVATIONS ARE DERIVATIONS}

In this section, the letter $\mathcal{L}$ still denotes an atomic Boolean subspace lattice on the Banach space $X$. But, $\mathcal{M} \subseteq \mathcal{B}(X)$ denotes an Alg $\mathcal{L}$-module, and $\delta$ a local derivation from $\operatorname{Alg} \mathcal{L}$ into $\mathcal{M}$. The purpose of this section is to prove that $\delta$ is in fact a derivation. Our proof follows by a modification of the arguments of Theorem 3.3 in [4]. Since, as remarked in Section 2, reflexive algebras with atomic Boolean invariant subspace lattices are strictly different from those reflexive algebras considered in [4], the proof is included here.

Let us begin with some lemmas. The first can be found in [4] which is the key to our results.

Lemma 4.1. Let $\delta$ be a local derivation from a Banach algebra $\mathcal{A}$ into an $\mathcal{A}$ module $\mathcal{U}$. Then $\delta(P A Q)=\delta(P A) Q+P \delta(A Q)-P \delta(A) Q$ holds for every $A \in \mathcal{A}$ and every pair $P, Q$ of idempotents in $\mathcal{A}$.

Lemma 4.2. For all $R_{1}, R_{2}$ in $\mathcal{R}_{\mathcal{L}}$ and all $A$ in $\operatorname{Alg} \mathcal{L}$, we have

$$
\delta\left(R_{1} A R_{2}\right)=\delta\left(R_{1} A\right) R_{2}+R_{1} \delta\left(A R_{2}\right)-R_{1} \delta(A) R_{2} .
$$

Proof: By Lemma 2.1, write $R_{1}=x \otimes f^{*}$ with $x \in K, f^{*} \in K_{-}^{\perp}$, and $R_{2}=y \otimes g^{*}$ with $y \in L, g^{*} \in L_{-}^{\perp}$, where $K$ and $L$ are two atoms of $\mathcal{L}$. It suffices to give the proof for the following three cases.

(1) Suppose $f^{*}(x) \neq 0$ and $g^{*}(y) \neq 0$. Let $R_{1}^{\prime}=R_{1} / f^{*}(x)$ and $R_{2}^{\prime}=R_{2} / g^{*}(y)$. Then both $R_{1}^{\prime}$ and $R_{2}^{\prime}$ are rank one idempotents, and hence we have $\delta\left(R_{1}^{\prime} A R_{2}^{\prime}\right)$ 
$=\delta\left(R_{1}^{\prime} A\right) R_{2}^{\prime}+R_{1}^{\prime} \delta\left(A R_{2}^{\prime}\right)-R_{1}^{\prime} \delta(A) R_{2}^{\prime}$ by Lemma 4.1. It follows from the linearity of $\delta$ that the equation $(*)$ holds.

(2) Suppose that precisely one of $f^{*}(x)$ and $g^{*}(y)$ is zero. Without loss of generality, assume that $f^{*}(x)=0$ and $g^{*}(y) \neq 0$. Since $K \cap K_{-}=(0), x \notin K_{-}$. So there exists a $f_{1}^{*} \in K_{-}^{\perp}$ such that $f_{1}^{*}(x) \neq 0$. Then $x \otimes f_{1}^{*}, x \otimes\left(f^{*}+f_{1}^{*}\right) \in \mathcal{R}_{\mathcal{L}}$. Thus we have by (1)

$$
\begin{aligned}
\delta\left(R_{1} A R_{2}\right)= & \delta\left(x \otimes\left(f^{*}+f_{1}^{*}\right) \cdot A \cdot y \otimes g^{*}\right)-\delta\left(x \otimes f_{1}^{*} \cdot A \cdot y \otimes g^{*}\right) \\
= & \delta\left(x \otimes\left(f^{*}+f_{1}^{*}\right) \cdot A\right) \cdot y \otimes g^{*}+x \otimes\left(f^{*}+f_{1}^{*}\right) \cdot \delta\left(A \cdot y \otimes g^{*}\right) \\
& -x \otimes\left(f^{*}+f_{1}^{*}\right) \cdot \delta(A) \cdot y \otimes g^{*}-\delta\left(x \otimes f_{1}^{*} \cdot A\right) \cdot y \otimes g^{*} \\
& -x \otimes f_{1}^{*} \cdot \delta\left(A \cdot y \otimes g^{*}\right)+x \otimes f_{1}^{*} \cdot \delta(A) \cdot y \otimes g^{*} \\
= & \delta\left(x \otimes f^{*} \cdot A\right) \cdot y \otimes g^{*}+x \otimes f^{*} \cdot \delta\left(A \cdot y \otimes g^{*}\right)-x \otimes f^{*} \cdot \delta(A) \cdot y \otimes g^{*} \\
= & \delta\left(R_{1} A\right) R_{2}+R_{1} \delta\left(A R_{2}\right)-R_{1} \delta(A) R_{2},
\end{aligned}
$$

as desired.

(3) Suppose that both $f^{*}(x)$ and $g^{*}(y)$ are zero. Since $K \cap K_{-}=L \cap L_{-}=(0)$, $x \notin K_{-}$and $y \notin L_{-}$. Then there exist $f_{1}^{*} \in K_{-}^{\perp}$ and $g_{1}^{*} \in L_{-}^{\perp}$ such that $f_{1}^{*}(x) \neq 0$ and $g_{1}^{*}(y) \neq 0$. Thus $x \otimes f_{1}^{*}, x \otimes\left(f^{*}+f_{1}^{*}\right), y \otimes g_{1}^{*}$ and $y \otimes\left(g^{*}+g_{1}^{*}\right) \in \mathcal{R}_{\mathcal{L}}$. Clearly, $\delta\left(R_{1} A R_{2}\right)=\delta\left(x \otimes\left(f^{*}+f_{1}^{*}\right) \cdot A \cdot y \otimes\left(g^{*}+g_{1}^{*}\right)\right)-\delta\left(x \otimes f_{1}^{*} \cdot A \cdot y \otimes\left(g^{*}+g_{1}^{*}\right)\right)-\delta\left(x \otimes f^{*} \cdot A \cdot y \otimes g_{1}^{*}\right)$.

By using (1) and (2), a routine computation similar to that which appeared in (2) implies the validity of the equation $(*)$. This concludes the proof.

Corollary 4.1. For all $R_{1}$ and $R_{2}$ in $\mathcal{R}_{\mathcal{L}}$, we have $\delta\left(R_{1} R_{2}\right)=\delta\left(R_{1}\right) R_{2}$ $+R_{1} \delta\left(R_{2}\right)$.

Proof: Since $\delta$ is a local derivation, $\delta(I)=0$. Taking $A=I$ in the equation (*), the desired result immediately follows and the proof is complete.

Lemma 4.3: ([10, Lemma 2.3].) Let $A \in \mathcal{B}(X)$. Then

(i) if $R A=0$ for every $R \in \mathcal{R}_{\mathcal{L}}$ then $A=0$;

(ii) if $A R=0$ for every $R \in \mathcal{R}_{L}$ then $A=0$.

LeMmA 4.4. For every $R \in \mathcal{R}_{\mathcal{L}}$ and every $A \in \operatorname{Alg} \mathcal{L}$, we have $\delta(A R)=\delta(A) R$ $+A \delta(R)$.

Proof: Let $R_{1} \in \mathcal{R}_{\mathcal{L}}$ be arbitrary. By Lemma 4.2 we have

$$
\delta\left(R_{1} A R\right)=\delta\left(R_{1} A\right) R+R_{1} \delta(A R)-R_{1} \delta(A) R .
$$

On the other hand, noting that $R_{1} A \in \mathcal{R}_{\mathcal{L}}$ if it is nonzero, then by Corollary 4.1

$$
\delta\left(R_{1} A R\right)=\delta\left(R_{1} A\right) R+R_{1} A \delta(R) .
$$

Equating these two equations we obtain $R_{1} \delta(A R)=R_{1} \delta(A) R+R_{1} A \delta(R)$. It follows from Lemma 4.3 (i) that $\delta(A R)=\delta(A) R+A \delta(R)$. This completes the proof. 
It is the time to prove the main result of this section.

THEOREM 4.1. Let $\mathcal{L}$ be an atomic Boolean subspace lattice on $X$, and $\mathcal{M}$ $\subseteq \mathcal{B}(X)$ be an $\operatorname{Alg} \mathcal{L}$-module. Then every local derivation $\delta$ from $\operatorname{Alg} \mathcal{L}$ into $\mathcal{M}$ is necessary a derivation.

Proof: Let $A, B \in \operatorname{Alg} \mathcal{L}$ be arbitrary. For any $R \in \mathcal{R}_{\mathcal{L}}$, we have by Lemma 4.4

$$
\begin{aligned}
\delta(A B) R+A B \delta(R) & =\delta(A B R)=\delta(A \cdot B R) \\
& =\delta(A) B R+A \delta(B R) \\
& =\delta(A) B R+A \delta(B) R+A B \delta(R) .
\end{aligned}
$$

Therefore $\delta(A B) R=\delta(A) B R+A \delta(B) R$. It follows from Lemma 4.3 (ii) that $\delta(A B)$ $=\delta(A) B+A \delta(B)$. This shows that $\delta$ is in fact a derivation, and the proof is complete.

In Theorem 4.1 letting $\mathcal{M}=\mathcal{B}(X)$, then

Corollary 4.2. Let $\mathcal{L}$ be an atomic Boolean subspace lattice on $X$. Then every local derivation from $\operatorname{Alg} \mathcal{L}$ into $\mathcal{B}(X)$ is a derivation.

From Theorem 3.1 and Theorem 4.1 we can obtain

Corollary 4.3. Let $\mathcal{L}$ be an atomic Boolean subspace lattice on $X$ and $\mathcal{M}$ be an ideal of $\operatorname{Alg} \mathcal{L}$. Then every local derivation from $\operatorname{Alg} \mathcal{L}$ into $\mathcal{M}$ is a quasi-spatial derivation.

In particular, we have

Corollary 4 .4. Let $\mathcal{L}$ be an atomic Boolean subspace lattice on $X$. Then every local derivation from $\operatorname{Alg} \mathcal{L}$ into itself is a quasi-spatial derivation.

\section{REFERENCEES}

[1] S. Argyros, M.S. Lambrou and W.E. Longstaff, 'Atomic Boolean subspace lattices and applications to the theory of bases', Mem. Amer. Math. Soc. 91 (1991).

[2] D. Han and S. Wei, 'Local derivations of nest algebras', Proc. Amer. Math. Soc. 123 (1995), 3095-3100.

[3] W. Jing, 'Local derivations of reflexive algebras', Proc. Amer. Math. Soc. 125 (1997), 869-873.

[4] W. Jing, 'Local derivations of reflexive algebras II', Proc. Amer. Math. Soc. 129 (2001), 1733-1737.

[5] B.E. Johnson, 'Local derivations on $\mathrm{C}^{*}$-algebras are derivations', Trans. Amer. Math. Soc. 353 (2001), 313-325.

[6] R.V. Kadison, 'Local derivations', J. Algebra 130 (1990), 494-509.

[7] A. Katavolos, M.S. Lambrou and W.E. Longstaff, 'Pentagon subspace lattices on Banach spaces', J. Operator Theory 46 (2001), 355-380.

[8] M.S. Lambrou, 'Approximants, commutants and double commutants in normed algebras', J. London Math. Soc. 225 (1982), 499-512. 
[9] M.S. Lambrou, On some reflexive lattices and related algebras, $\mathrm{Ph}$. D. dissertation (University of London, Kings College, 1977).

[10] M.S. Lambrou, 'On the rank of operators in reflexive algebras', Linear Algebra Appl. 142 (1990), 211-235.

[11] D.R. Larson and A.R. Sourour, 'Local derivations and local automorphisms of $B(X)$ ', Proc. Sympos. Pure Math. 51 (1990), 187-194.

[12] W.E. Longstaff, 'Strongly reflexive lattices', J. London Math. Soc. (2) 11 (1975), 491-498.

[13] O. Panaia, 'Algebraic isomorphisms and finite distributive subspace lattices', $J$. London Math. Soc. (2) 59 (1999), 1033-1048.

Department of Mathematics

Nanjing University

Nanjing

Jiangsu 210093

People's Republic of China

e-mail: ptli@nju.edu.cn

pengtonglee@sina.com.cn
Department of Mathematics

Nanjing University

Nanjing

Jiangsu 210093

People's Republic of China 